\title{
Some Algebraic and Analytical Properties of Coquaternion Algebra
}

\author{
Anatoliy A. Pogoruy and Ramón M. Rodríguez-Dagnino
}

\begin{abstract}
The aim of this work is to prove some basic properties of coquaternions and to find the zeros of polynomials involving coquaternionic elements. We also provide some expansions of hyperholomorphic function on coquaternion algebra by using Fueter polynomials.
\end{abstract}

Mathematics Subject Classification (2000). Primary 37F10; Secondary 32A10. Keywords. Coquaternion algebra, zeros of quaternionic polynomials, hyperholomorphic function, Fueter polynomials.

\section{Introduction to Coquaternions}

Let $\mathbb{C}$ be the complex field, and let $\mathbb{A} \mathbb{H}=\left\{a_{0}+a_{1} i+a_{2} e+a_{3} f\right\}$ be the coquaternion algebra over the real field $\mathbb{R}$, where $a_{0}, a_{1}, a_{2}, a_{3} \in \mathbb{R}, i^{2}=-1, e^{2}=f^{2}=1$, $i e=-e i=f, f i=-i f=e$, and $e f=-f e=-i$.

Coquaternions were initially introduced by James Cockle [1] and they are also called para-quaternions [2] or anti-quaternions [3].

The conjugate of $w=a_{0}+a_{1} i+a_{2} e+a_{3} f$ is defined as $\bar{w}=a_{0}-a_{1} i-a_{2} e-a_{3} f$. Thus $w \bar{w}=\bar{w} w=a_{0}^{2}+a_{1}^{2}-a_{2}^{2}-a_{3}^{2}$. The module $|w|$ of $w$ is defined as $\sqrt{w \bar{w}}$. When $w \bar{w}<0$ then the value of the module is a complex number from the upper half of the complex plane [3]. Coquaternions are also named split-quaternions because of the special signature of the quadratic form defined by their modules.

The coquaternion algebra is an associative and noncommutative 4-dimensional Clifford algebra $[1,3]$.

Similarly to the quaternions notation we will denote by $S c(w)=\frac{w+\bar{w}}{2}$ and $\operatorname{Vec}(w)=\frac{w-\bar{w}}{2}$ the scalar and vector part of $w$ respectively. The following

This work was completed with the support of the State Fund to Fundamental researches of Ukraine, Project No. 25, 1/084, and the Chair on Telecommunications at Tecnológico de Monterrey. 
properties can be checked by routine calculations: For any $v, w \in \mathbb{A} \mathbb{H}$ we have that $\overline{v+w}=\bar{v}+\bar{w}$ and $\overline{w v}=\bar{v} \bar{w}$. From these properties, it follows that $v w \overline{v w}=v w \bar{w} \bar{v}$, hence $|v w|^{2}=|v|^{2}|w|^{2}$.

Now, let $c_{1}, c_{2}$ be complex numbers and $w$ be a coquaternion. It is easy to see that any coquaternion $w$ can be represented in the form $c_{1}+c_{2} e$. Indeed, $a_{0}+a_{1} i+a_{2} e+a_{3} f=\left(a_{0}+a_{1} i\right)+\left(a_{2}+a_{3} i\right) e$.

\section{On the Set of Zeros of Coquaternion Polynomials}

Let us denote as $Z(\mathbb{A} \mathbb{H})$ the set of zero divisors of algebra $\mathbb{A} \mathbb{H}$. The following lemma can be easily proved

Lemma 2.1. $w \in Z(\mathbb{A} \mathbb{H})$ iff $|w|=0$.

Let us introduce an equivalence relation $\sim$ over $\mathbb{A} \mathbb{H}$ as follows: For any two coquaternions $w$ and $w^{\prime}, w \sim w^{\prime}$ if there exists $x \in \mathbb{A} \mathbb{H} \backslash Z(\mathbb{A} \mathbb{H})$ such that $w^{\prime}=$ $x^{-1} w x$. The congruence class of $w$, denoted by $[w]$, is the set $\{v \in \mathbb{A} \mathbb{H}: v \sim w\}$.

Lemma 2.2. For any $w \in \mathbb{A} \mathbb{H},[w]$ is the set $[w]=\{v \in \mathbb{A} \mathbb{H}: S c(v)=S c(w)$ and $\left.|v|^{2}=|w|^{2}\right\}$.

Proof. Suppose that $w=a_{0}+a_{1} i+a_{2} e+a_{3} f$ and $v=b_{0}+b_{1} i+b_{2} e+b_{3} f$ belong to the congruence class $[w]$. Then, there exists $x=x_{0}+x_{1} i+x_{2} e+x_{3} f \in \mathbb{A} \mathbb{H} \backslash Z(\mathbb{A} \mathbb{H})$ such that

$$
w x=x v .
$$

Comparing coefficients of $i, e$ and $f$ in Eq. (2.1), we get

$$
\begin{aligned}
& \left(a_{0}-b_{0}\right) x_{0}+\left(b_{1}-a_{1}\right) x_{1}+\left(a_{2}-b_{2}\right) x_{2}+\left(a_{3}-b_{3}\right) x_{3}=0 \\
& \left(a_{1}-b_{1}\right) x_{0}+\left(a_{0}-b_{0}\right) x_{1}+\left(a_{3}+b_{3}\right) x_{2}-\left(a_{2}+b_{2}\right) x_{3}=0 \\
& \left(a_{2}-b_{2}\right) x_{0}+\left(a_{3}+b_{3}\right) x_{1}+\left(a_{0}-b_{0}\right) x_{2}-\left(a_{1}+b_{1}\right) x_{3}=0 \\
& \left(a_{3}-b_{3}\right) x_{0}-\left(a_{2}+b_{2}\right) x_{1}+\left(a_{1}+b_{1}\right) x_{2}+\left(a_{0}-b_{0}\right) x_{3}=0 .
\end{aligned}
$$

The system of equations (2.2) has a nontrivial solution iff

$$
\operatorname{det}\left(\begin{array}{llll}
a_{0}-b_{0} & b_{1}-a_{1} & a_{2}-b_{2} & a_{3}-b_{3} \\
a_{1}-b_{1} & a_{0}-b_{0} & a_{3}+b_{3} & -a_{2}-b_{2} \\
a_{2}-b_{2} & a_{3}+b_{3} & a_{0}-b_{0} & -a_{1}-b_{1} \\
a_{3}-b_{3} & -a_{2}-b_{2} & a_{1}+b_{1} & a_{0}-b_{0}
\end{array}\right)=0 .
$$

Eq. (2.3) is fulfilled iff $a_{0}=b_{0}$ and $a_{1}^{2}-a_{2}^{2}-a_{3}^{2}=b_{1}^{2}-b_{2}^{2}-b_{3}^{2}$.

Let us consider the following polynomial of coquaternionic variable $w$ :

$$
p_{n}(w)=a_{n} w^{n}+a_{n-1} w^{n-1}+\cdots+a_{0},
$$

where $a_{k} \in \mathbb{A} \mathbb{H}$ for $k=1,2, \ldots, n$.

Since $w$ is a zero of the quadratic equation with real coefficients

$$
w^{2}-p w+q=0
$$


where $p=2 S c(w)=w+\bar{w}, q=|w|^{2}=w \bar{w}$, we have $w^{2}=p w-q$. Hence, $w^{3}=p w^{2}-q w=\left(p^{2}-q\right) w-p q$. Continuing with the same procedure we get

$$
w^{n}=F_{n}(w) w+G_{n}(w),
$$

where $F_{n}(w)$ and $G_{n}(w)$ are real-valued functions defined by the recurrent formulae

$$
F_{n+1}(w)=p F_{n}(w) w+G_{n}(w)=p F_{n}(w)-q F_{n-1}(w)
$$

and

$$
G_{n+1}(w)=-q F_{n}(w),
$$

with $F_{1}(w)=1, F_{2}(w)=w+\bar{w}, G_{1}(w)=0$ and $G_{2}(w)=-|w|^{2}$.

Therefore, the polynomial (2.4) can be represented in the following form

$$
p_{n}(w)=F(w) w+G(w),
$$

where $F(w)=\sum_{n} a_{n} F_{n}(w)$ and $G(w)=\sum_{n} a_{n} G_{n}(w)$.

Lemma 2.3. For any equivalent coquaternions $w_{0} \sim w_{1}$, where $w_{0}, w_{1} \in \mathbb{A} \mathbb{H}$, we have

$$
\begin{aligned}
& F\left(w_{0}\right)=F\left(w_{1}\right), \\
& G\left(w_{0}\right)=G\left(w_{1}\right) .
\end{aligned}
$$

Proof. The functions $F_{n}(w)$ and $G_{n}(w)$ depend on $S c(w)$ and the norm of $w$. Then, it follows from Lemma 2.2 that $F_{n}\left(w_{0}\right)=F_{n}\left(w_{1}\right)$ and $G_{n}\left(w_{0}\right)=G_{n}\left(w_{1}\right)$ for any $n \geq 1$. From these results, it follows that $F\left(w_{0}\right)=F\left(w_{1}\right)$ and $G\left(w_{0}\right)=G\left(w_{1}\right)$.

Theorem 2.4. Assume that $w_{0} \in \mathbb{A} \mathbb{H}$, and $\operatorname{Vec}\left(w_{0}\right)$ is not a zero divisor. If $w_{1} \sim w_{0}$ and $w_{0}, w_{1}$ are different zeroes of polynomial (2.4), then any coquaternion $w \in\left[w_{0}\right]$ is a zero of (2.4). So, we might say that the congruence class of $w$ is a zero of polynomial (2.4).

Proof. By using Eq. (2.5) and Lemma 2.4, we have

$$
\begin{aligned}
& p_{n}\left(w_{0}\right)=F\left(w_{0}\right) w_{0}+G\left(w_{0}\right)=0, \\
& p_{n}\left(w_{1}\right)=F\left(w_{0}\right) w_{1}+G\left(w_{0}\right)=0 .
\end{aligned}
$$

Hence,

$$
F\left(w_{0}\right)\left(w_{0}-w_{1}\right)=0 .
$$

Since $\operatorname{Vec}\left(w_{0}\right)$ is not a zero divisor then the coquaternion $w_{0}-w_{1}$ cannot be a zero divisor. Indeed, let $w_{0}=a_{0}+a_{1} i+a_{2} e+a_{3} f$ and $w_{1}=a_{0}+b_{1} i+b_{2} e+b_{3} f$. Since $w_{0} \sim w_{1}$ we have $a_{1}^{2}-a_{2}^{2}-a_{3}^{2}=b_{1}^{2}-b_{2}^{2}-b_{3}^{2} \neq 0$, i.e., points $A\left(a_{1}, a_{2}, a_{3}\right)$ and $B\left(b_{1}, b_{2}, b_{3}\right)$ lie on a hyperboloid. Suppose that $w_{0}-w_{1}$ is a zero divisor, then it follows from Lemma 2.2 that the point $C\left(a_{1}-b_{1}, a_{2}-b_{2}, a_{3}-b_{3}\right)$ lies on the asymptotic cone $x_{1}^{2}-x_{2}^{2}-x_{3}^{2}=0$ of the hyperboloid, but this is impossible. In this case, it follows from Eq. (2.6) that $F\left(w_{0}\right)=0$. Therefore, $G\left(w_{0}\right)=0$ and any $w \in\left[w_{0}\right]$ is a zero of polynomial (2.4). 
A set of polynomial zeros $\left[w_{0}\right]$ will be called a hyperboloidal zero.

It is easy to see that the polynomial $p_{n}(w)=w^{n} a_{n}+w^{n-1} a_{n-1}+\cdots+a_{0}$ can be investigated much in a similar manner.

Theorem 2.5. Suppose coefficients $r_{k}, k=0,1, \ldots, n$ of a polynomial over $\mathbb{A} \mathbb{H}$ $p_{n}(w)=r_{n} w^{n}+r_{n-1} w^{n-1}+\cdots+r_{0}$ are real numbers then all non-real zeros of the polynomial $p_{n}(w)$ are hyperboloidal.

Proof. Suppose $w_{0}$ is a zero of $p_{n}(w)$, then it is easily verified that for any $x \in$ $\mathbb{A} \mathbb{H} \backslash Z(\mathbb{A} \mathbb{H}):$

$$
r_{n}\left(x^{-1} w_{0} x\right)^{n}+r_{n-1}\left(x^{-1} w_{0} x\right)^{n-1}+\cdots+r_{1}\left(x^{-1} w_{0} x\right)+r_{0}=0 .
$$

Since a complex number $a+b i$ belongs to $[w]$, where $w=a+a_{1} i+a_{2} e+a_{3} f$ with $a_{1}^{2}-a_{2}^{2}-a_{3}^{2}=b^{2}$, and the theorem is proved.

The set of zeros of quaternionic polynomials was studied in [4].

\section{Hyperholomorphic Functions on Coquaternion Algebra}

Let us consider the following Cauchy-Fueter type of operator for coquaternions

$$
D:=\frac{\partial}{\partial x_{0}}+i \frac{\partial}{\partial x_{1}}+e \frac{\partial}{\partial x_{2}}+f \frac{\partial}{\partial x_{3}} .
$$

Similarly to Fueter ideas [5], we determine a hyperholomorphic function on $\mathbb{A} \mathbb{H}$ as follows:

A function $g: \mathbb{R}^{4} \longrightarrow \mathbb{A} \mathbb{H}$ is called left-hyperholomorphic or left-monogenic if it satisfies the equation $D g=0$. A right-hyperholomorphic function $g$ is the solution of the equation $g D=0$. As in the case of quaternion algebra the set of hyperholomorphic functions on the coquaternion algebra does not contain polynomials. To overcome these difficulties we consider the so-called Fueter polynomials

$$
\varsigma_{1}(x)=x_{1}-i x_{0}, \quad \varsigma_{2}(x)=x_{2}-e x_{0}, \quad \varsigma_{3}(x)=x_{3}-f x_{0} .
$$

It is easy to show that Fueter polynomials are both right and left hyperholomorphic.

Lemma 3.1. Suppose $g$ is a left-hyperholomorphic function then for any real $t$ we have

$$
\frac{d g}{d t}(t x)=\sum_{k=1}^{3} \varsigma_{k}(x) \frac{\partial g}{\partial x_{k}}(t x) .
$$

Proof. Since $g$ is left-hyperholomorphic we have

$$
\frac{\partial}{\partial x_{0}} g=-i \frac{\partial}{\partial x_{1}} g-e \frac{\partial}{\partial x_{2}} g-f \frac{\partial}{\partial x_{3}} g .
$$

By plugging this expression into

$$
\frac{d g}{d t}(t x)=\sum_{k=0}^{3} x_{k} \frac{\partial g}{\partial x_{k}}(t x)
$$


the proof of the lemma is achieved.

Now, it follows from Eq. (3.1) that

$$
g(x)=g(0)+\sum_{k=1}^{3} \varsigma_{k}(x) \int_{0}^{1} \frac{\partial g}{\partial x_{k}}(t x) d t .
$$

Let us assume that $g(x)=u_{0}(x)+u_{1}(x) i+u_{2}(x) e+u_{3}(x) f$, where $u_{k}(x) \in$ $\mathcal{C}^{\infty}$. Thus, it is easy to verify that the function $m(x):=\int_{0}^{1} \frac{\partial g}{\partial x_{k}}(t x) d t$ is lefthyperholomorphic as well. Thus, by applying Eq. (3.2) to $m(x)$, we obtain

$$
g(x)=g(0)+\sum_{k=1}^{3} \varsigma_{k}(x) \frac{\partial g}{\partial x_{k}}(0)+\sum_{l=1}^{3} \sum_{k=1}^{3}\left(\varsigma_{l}(x) \varsigma_{k}(x)\right) \int_{0}^{1} \int_{0}^{1} \frac{\partial^{2} g}{\partial x_{l} \partial x_{k}}(s t x) d t d s,
$$

where $s, t \in \mathbb{R}$.

By iterating this procedure, we can expand the function $g(x)$ in terms of products of Fueter polynomials

$$
\begin{aligned}
g(x) & =g(0)+\sum_{k=1}^{3} \varsigma_{k}(x) \frac{\partial g}{\partial x_{k}}(0)+\sum_{l=1}^{3} \sum_{k=1}^{3} \varsigma_{l}(x) \varsigma_{k}(x) \frac{\partial^{2} g}{\partial x_{l} \partial x_{k}}(0)+\cdots \\
& +\sum_{j_{1}=1}^{3} \sum_{j_{2}=1}^{3} \cdots \sum_{j_{n}=1}^{3} \varsigma_{j_{1}}(x) \varsigma_{j_{2}}(x) \cdots \varsigma_{j_{n}}(x) \frac{\partial^{n} g}{\partial x_{j_{1}} \partial x_{j_{2}} \cdots \partial x_{j_{n}}}(0)+\cdots .
\end{aligned}
$$

Denote by $\mathcal{S}$ the set

$$
\mathcal{S}=\left\{x \in \mathbb{A} \mathbb{H}:|x| \lim _{n \rightarrow \infty} \sqrt[n]{\sup _{j_{1}, \ldots, j_{n}}\left|\frac{\partial^{n} g}{\partial x_{j_{1}} \partial x_{j_{2}} \cdots \partial x_{j_{n}}}(0)\right|} \leq c<\frac{1}{3}\right\} .
$$

Hence, it is easy to show that for $x \in \mathcal{S}$ the series in Eq. (3.3) is convergent. As a consequence of this fact we have that if a polynomial is left-hyperholomorphic then it has to be of the following form

$$
p_{n}(x)=\sum_{k=1}^{n}\left(\sum_{j_{1}=1}^{3} \sum_{j_{2}=1}^{3} \cdots \sum_{j_{k}=1}^{3} \varsigma_{j_{1}}(x) \varsigma_{j_{2}}(x) \cdots \varsigma_{j_{k}}(x)\right) a_{j_{1} j_{2} \ldots j_{k}} .
$$

In the particular case when $a_{j_{1} j_{2} \ldots j_{k}}=a_{k}$ for all $j_{1}, j_{2}, \ldots, j_{k} \in\{1,2,3\}$ we have

$$
p_{n}(x)=\sum_{k=1}^{n}\left(\varsigma_{1}(x)+\varsigma_{2}(x)+\varsigma_{3}(x)\right)^{k} a_{k} .
$$

Remark 3.2. An expansion of hyperholomorphic quaternion functions in Fueter polynomials has been reported in [6]. 


\section{Conclusions}

The mapping $w^{\prime}=x^{-1} w x$ in coquaternionic algebra represents a hyperbolic rotation and has a tight relationship to the Lorentz group used in relativity theory. So, coquaternions are interesting not only from the mathematical point of view but also from their applications in modern physics [7]. In spite of their importance, the problem of finding the roots of coquaternionic polynomials and the notion of hyperholomorphic function and its properties have not been reported in the literature. So far, in most of the papers dealing with coquaternions the authors are limited to mention just a few of their basic properties and the possible application of this algebra to geometry and physics. In this paper, we investigated the set of zeros of one-sided polynomials, and by following the ideas of Fueter, we have introduced the notion of hyperholomorphic function or monogenic function and we provide an expansion of such functions in series of Fueter polynomials.

\section{References}

[1] J. Cockle, On Systems of Algebra involving more than one Imaginary. Philosophical Magazine 35 (3) (1849), 434-435.

[2] T. Mohaupt, New developments in Special Geometry. arXiv:hep-th/0602171v1 (2006).

[3] B. A. Rosenfeld, Many-dimensional Geometries. Gostehizdat, Moscow (1966), in Russian.

[4] A. A. Pogorui and M. Shapiro, On the structure of the Set of Zeros of Quaternionic Polynomials. Complex Variables 49 (6) (2004), 379-389.

[5] R. Fueter, Analitische Funktionen einer Quaternionen variablen. Comment. Math. Helv. 4 (1932), 9-20.

[6] D. Alpay and M. Shapiro, Problème de Gleason et interpolation pour les fonctions hyper-analytiques. C. R. Acad. Sci. Paris, Ser. I 335 (2002), 1-6.

[7] N. Blaz̄ié, Paraquaternionic projective space and pseudo-Riemannian geometry. Publications de L'Institut Mathèmatique, Nouvelle sèries, tome 60 (74), (1996), 101-107.

Anatoliy A. Pogoruy

Department of Mathematics

Zhytomyr State University

Zhytomyr, 10008, Ukraine

e-mail: pogor@zu.edu.ua

Ramón M. Rodríguez-Dagnino

Tecnológico de Monterrey

E. Garza Sada 2501 Sur, Col. Tecnológico, C.P. 64849

Monterrey, NL, México

e-mail: rmrodrig@itesm.mx

Received: January 7, 2008.

Accepted: August 06, 2008. 